\title{
Analysis of Thermal Damage in a Laser-Irradiated Based on the Non-Fourier Model
}

\author{
Kuo-Chi Liu, Po-Jen Cheng, and Jiun-Shen Wang
}

\begin{abstract}
A modified non-Fourier equation of bio-heat transfer based on the second-order Taylor expansion of dual-phase-lag model is proposed to estimating thermal damage in a laser-irradiated biological tissue. This non-Fourier bio-heat transfer equation involves the mixed-derivative terms and the high-order derivatives of temperature with respect to time. The thermal damage in the tissue is assessed with the rate process equation. The effects of blood perfusion and metabolic heat generation on thermal response and thermal damage are explored. There are mathematical difficulties in dealing with such a problem. A hybrid numerical scheme is extended to solve the present problem. The deviations of the results from the bio-heat transfer equation based on the linear form of the dual-phase-lag model are presented and discussed.
\end{abstract}

Index Terms-Bio-heat transfer, dual-phase-lag mode, laplace transform, scattering tissue.

\section{INTRODUCTION}

The Pennes bio-heat equation describes the thermal behavior based on the classical Fourier's law which depicts an infinitely fast propagation of thermal signal. In reality, the living tissues are highly non-homogenous and need a relaxation time to accumulate enough energy to transfer to the nearest element. As a result, to solve the paradox occurred in the Pennes model, the non-Fourier models of bio-heat transfer were proposed for the investigation of physical mechanisms and the behaviors in thermal propagation in living tissues [1,2]. In order to consider the effect of micro-structural interactions in the fast transient process of heat transport, a phase lag for temperature gradient absent in the thermal wave model was introduced [3]. The corresponding model is called the dual-phase-lag (DPL) model. Antaki [2] used the DPL model to interpret heat conduction in processed meat that was interpreted with the thermal wave model. $\mathrm{Xu}$ et al. [4] presented a system discussion on the application of the DPL model in the biothermomechanical behavior of skin tissue. Liu and his co-workers $[5,6]$ did an extension study for exploring whether the DPL thermal behavior exists in tissue.

The heat transfer equation used in the above papers was developed with the linear form of the DPL model involving

Manuscript received June 9, 2013; revised July 20, 2013. This work was supported in part by the National Science Council in Taiwan under Grant NSC 100-2221-E-269-015 and NSC 101-2221-E-269-006.

Kuo-Chi Liu and Po-Jen Cheng are with the Department of Mechanical Engineering, Far East University, No 49 Chung Hua Rd., Hsin-Shih, Tainan 74448, Taiwan. (e-mail: kcliu@cc.feu.edu.tw; pjcheng@cc.feu.edu.tw).

Jiun-Shen Wang is with the Department of Power Mechanical Engineering, National Taitung College, No.889, Jhengci N. Rd., Taitung, 95045 Taiwan (email: wjs99@ntc.edu.tw) the linear effects of the phase lag times. Recently, the non-linear effects of the phase lag times on transport phenomena were introduced for multi-carrier [7], [8]. The more rational prediction of temperature distribution is always needed in the development of thermal medical treatments. For a more general form, this paper develops a modified DPL equation of bio-heat transfer based on the second-order Taylor expansion. For convenience of statement, this paper calls it non-linear DPL equation. The non-linear DPL equation is a fourth order partial differential equation. There are mathematical difficulties in dealing with such an equation. The hybrid numerical scheme [9] based on the Laplace transform and the modified discretization technique is extended to solve the present problem.

\section{PROBlem Formulation}

In order to solve the paradox occurred in the classical heat flux model and to consider the effect of micro-structural interactions, the DPL model was suggested [3] with

$$
\vec{q}\left(t+\tau_{q}\right)=-k \nabla T\left(t+\tau_{T}\right)
$$

where $T$ is the temperature, $k$ the heat conductivity, $q$ the heat flux, and $t$ the time. $\tau_{q}$ means the phase lag of the heat flux and $\tau_{T}$ means the phase lag of the temperature gradient.

Equation (1) is, usually, developed in the first-order Taylor series expansion. As the literature [4] did, this paper would develop (1) in the second-order Taylor series expansion for a more general form. Thus, it is rewritten as

$$
\left(1+\tau_{q} \frac{\partial}{\partial t}+\frac{\tau_{q}^{2}}{2} \frac{\partial^{2}}{\partial t^{2}}\right) \vec{q}=-\left(1+\tau_{T} \frac{\partial}{\partial t}+\frac{\tau_{T}^{2}}{2} \frac{\partial^{2}}{\partial t^{2}}\right) k \nabla T
$$

In a local energy balance, the energy conservation equation of bio-heat transfer is described as

$$
\rho C \frac{\partial T}{\partial t}=-\nabla \cdot \vec{q}+w_{b} \rho_{b} c_{b}\left(T_{b}-T\right)+q_{m}+q_{r}
$$

where $t$ is time. $\rho, c$, and $T$ denote density, specific heat, and temperature of tissue. $c_{b}$ and $w_{b}$ are, respectively, the specific heat and perfusion rate of blood. $q_{m}$ is the metabolic heat generation and $q_{r}$ is the heat source for spatial heating. $T_{b}$ is the arterial temperature.

Substituting (2) into the energy conservation (3) leads to the second-order DPL equation of bio-heat transfer with constant physiological parameters as the following: 


$$
\begin{aligned}
& \left(1+\tau_{T} \frac{\partial}{\partial t}+\frac{\tau_{T}^{2}}{2} \frac{\partial^{2}}{\partial t^{2}}\right) k \nabla^{2} T \\
& =\left(1+\tau_{q} \frac{\partial}{\partial t}+\frac{\tau_{q}^{2}}{2} \frac{\partial^{2}}{\partial t^{2}}\right)\left[\rho c \frac{\partial T}{\partial t}-w_{b} \rho_{b} c_{b}\left(T_{b}-T\right)-q_{m}-q_{r}\right]
\end{aligned}
$$

As $\tau_{T}=0$, equation (4) undergoes to the hyperbolic heat transfer equation with negating second-order Taylor series expansion for $\tau_{q}$. Deleting the second-order Taylor series expansions for $\tau_{q}$ and $\tau_{T}$ leads to the first-order DPL equation of bio-heat transfer. On the other hand, equation (4) would reduce to the Pennes equation for $\tau_{q}=\tau_{T}=0$.

Consider a broad laser beam with a uniform irradiance $\left(\phi_{i n}\right)$ is applied normally to a finite slab of biological tissue with a thickness of $L$ at time $t=0^{+}$. When the spot size of the broad laser beam is much larger than the thickness of the thermally affected zone for the time period of interest, a 1-D model would be sufficient for analyzing the thermal response of the heated medium [10], [11].

The 1-D form of (4) with constant thermal parameters is written as

$$
\begin{aligned}
& \left(1+\tau_{T} \frac{\partial}{\partial t}+\frac{\tau_{T}^{2}}{2} \frac{\partial^{2}}{\partial t^{2}}\right) k \frac{\partial^{2} T}{\partial x^{2}} \\
& =\left(1+\tau_{q} \frac{\partial}{\partial t}+\frac{\tau_{q}^{2}}{2} \frac{\partial^{2}}{\partial t^{2}}\right)\left[\rho c \frac{\partial T}{\partial t}-w_{b} \rho_{b} c_{b}\left(T_{b}-T\right)-q_{m}-q_{r}\right]
\end{aligned}
$$

When laser irradiation is strongly scattered in the tissue, laser heating serves as a spatially-varied body heat source that heats up the tissue. Under broad beam irradiance, the light distribution in scattering tissues is described with two exponentials as [12]

$$
\phi(x)=\phi_{i n}\left[C_{1} \exp \left(-d_{1} x / \delta\right)-C_{2} \exp \left(-d_{2} x / \delta\right)\right]
$$

where the coefficients $C_{1}, d_{1}, C_{2}, d_{2}$ depend on the diffuse reflectance and can be obtained from the Monte Carlo solutions, ; $\delta$ the effective optical penetration depth, which is defined based on the diffusion theory as

$$
\delta=\frac{1}{\sqrt{3 \mu_{a}\left[\mu_{a}+\mu_{s}(1-g)\right.}}
$$

where $\mu_{a}$ is the absorption coefficient and $\mu_{s}$ is the scattering coefficient. The reduced scattering coefficient $\mu_{s}^{\prime}$ is introduced as $\mu_{s}^{\prime}=\mu_{s}(1-g)$, in which $g$ is the anisotropy factor.

Therefore, the laser volumetric heat source $q_{r}$ can be described as

$$
q_{r}=\mu_{a} \phi(x)
$$

This study assumes the laser irradiance $\phi_{\text {in }}$ is deposited as

$$
\phi_{\text {in }}=q_{0}[u(t)-u(t-\Delta t)]
$$

where $q_{0}$ is the laser intensity, $\Delta t$ is the laser exposure time, and $u$ is step function.

For strongly scattering tissues, laser heating is considered as a body heat source and the irradiated surface is regarded as being thermally insulated. The boundary conditions for this case thus become [10]

$$
q=0 \text { for } x=0, L \text { when } t>0
$$

The studied problem has the initial conditions

$$
T(x, 0)=T_{b} \text { and } \frac{\partial T(x, 0)}{\partial t}=\frac{\partial^{2} T(x, 0)}{\partial t^{2}}=0
$$

For estimation of thermal damage in a living tissue, the damage parameter $\Omega$ was defined as

$$
\Omega=\ln \left(\frac{C_{0}}{C_{0}-C_{d}}\right)
$$

where $C_{0}$ is the protein concentration in the normal tissue, $C_{d}$ the concentration of denaturated protein. Welch [13] regarded the protein denaturation process as a chemical reaction. The Arrenius equation was used to evaluate the damage parameter:

$$
\Omega=A \int_{t_{1}}^{t_{f}} \exp \left(-\frac{E}{R T}\right) d t
$$

where $A$ is the frequency factor; $E$ the energy of activation of denaturation reaction; $R$ the universal gas constant, 8.314 $\mathrm{J} /(\mathrm{mol} \mathrm{K}) ; T$ the absolute temperature of the tissue at the point where $\Omega$ is calculated; $t_{1}$ the time at the onset of laser exposure; and $t_{f}$ the time when the thermal damage is evaluated [10].

When the protein denaturation reaches $63 \%$ of the molecules, which corresponds to $\Omega=1.0$, the tissue is assumed to be irreversible damaged. The values of the frequency factor and the activation energy are given as: $A=$ $3.1 \times 10^{98} \mathrm{~s}-1 ; E=6.28 \times 10^{5} \mathrm{~J} / \mathrm{mol}[13]$.

\section{ANALyticAl MethoD}

For a brief statement, the elevation variable $H$ is defined as $H=T-T_{b}$. As a result, Equation (4) is rewritten as

$$
\begin{aligned}
& k\left(1+\tau_{T} \frac{\partial}{\partial t}+\frac{\tau_{T}^{2}}{2} \frac{\partial^{2}}{\partial t^{2}}\right) \frac{\partial^{2}}{\partial x^{2}} \\
& =\left(1+\tau_{q} \frac{\partial}{\partial t}+\frac{\tau_{q}^{2}}{2} \frac{\partial^{2}}{\partial t^{2}}\right)\left(\rho c \frac{\partial H}{\partial t}+w_{b} \rho_{b} c_{b} H-q_{m}-q_{r}\right)
\end{aligned}
$$

The initial conditions are also rewritten as

$$
H(x, 0)=0 \text { and } \frac{\partial H(x, 0)}{\partial t}=\frac{\partial^{2} H(x, 0)}{\partial t^{2}}=0
$$

First, the Laplace transform method is employed to map 
the transient problem into steady one. Equation (14) and the boundary conditions can be written in the transform domain based on the initial conditions (15) as

$$
\begin{gathered}
\frac{d^{2} \tilde{H}}{d x^{2}}-\lambda^{2} \tilde{H}=-g \\
\frac{d \tilde{H}}{d x}=0 \quad \text { for } x=0, L
\end{gathered}
$$

where

$$
\begin{gathered}
\lambda^{2}=\frac{1}{K}\left[\rho c s+w_{b} \rho_{b} c_{b}\right] \\
K=\frac{k\left(1+\tau_{T} s+\tau_{T}^{2} s^{2} / 2\right)}{1+\tau_{q} s+\tau_{q}^{2} s^{2} / 2} \\
g=\frac{1}{k\left(1+\tau_{T} s+\tau_{T}^{2} s^{2} / 2\right)}\left[\frac{q_{m}}{s}+\widetilde{q}_{r}\left(1+\tau_{q} s+\tau_{q}^{2} s^{2} / 2\right)\right]
\end{gathered}
$$

The function $H$ is written as $\widetilde{H}$ in the Laplace domain. $s$ is the Laplace transform parameter with respect to $t$.

A modified discretization scheme is applied to numerically solve the present problem. Please refer to the literature [9] for the details of the present numerical scheme.

\section{RESUlTS AND Discussion}

The values of relevant thermal parameters in the calculations are taken from the paper [10]. The thermalphysical properties of tissue are: $k=0.628 \mathrm{~W} / \mathrm{m} \cdot \mathrm{K}$, $\rho=1000 \mathrm{~kg} / \mathrm{m}^{3}$, and $c=4187 \mathrm{~J} /(\mathrm{kg} \cdot \mathrm{K})$. The thermalphysical properties and temperature of blood are: $\rho_{b}=1.06 \times 10^{3}$ $\mathrm{kg} / \mathrm{m}^{3}, c_{b}=3860 \mathrm{~J} /(\mathrm{kg} \cdot \mathrm{K}), w_{b}=1.87 \times 10^{-3} \mathrm{~m}^{3} /\left(\mathrm{m}^{3} \cdot \mathrm{s}\right), T_{b}=37$ ${ }^{\circ} \mathrm{C}$. The metabolic heat generation is $q_{m}=1.19 \times 10^{3} \mathrm{~W} / \mathrm{m}^{3}$. The optical properties are: $\mu_{a}=0.4 \mathrm{~cm}^{-1}, \mu_{s}=120 \mathrm{~cm}^{-1}, \mathrm{~g}$ $=0.9$, and $R_{d}=0.05$. The values of $L$ and $\Delta t$ are specified as 5 $\mathrm{cm}$ and $5 \mathrm{~s}$, respectively. The incident laser irradiance is considered, $q_{0}=30 \mathrm{~W} / \mathrm{cm}^{2}$. The relaxation times are specified as $\tau_{q}=15.5 \mathrm{~s}, \tau_{T}=10 \mathrm{~s}$.

$\mathrm{T}$ The adjustment of temperature is one of the functions of blood circulation in a living tissue. The effect of the perfusion rate on the bio-heat transfer is needed to pay attention. The effects of the blood perfusion rate on the temperature and thermal damage are demonstrated in Fig. 1. The blood perfusion develops a cooling function, since the tissue temperature is higher than the arterial temperature. At the initial times, the heat energy carried off by the perfusion blood is small. Therefore, the surface temperatures for $w_{b}=$ $1.87 \times 10^{-3} \mathrm{~m}^{3} /\left(\mathrm{m}^{3} \cdot \mathrm{s}\right), w_{b}=2.8 \times 10^{-3} \mathrm{~m}^{3} /\left(\mathrm{m}^{3} \cdot \mathrm{s}\right)$ and $w_{b}=$ $3.8 \times 10^{-3} \mathrm{~m}^{3} /\left(\mathrm{m}^{3} \cdot \mathrm{s}\right)$ do not have obvious difference. After that, the difference increases with time for the cooling function of the blood perfusion. The heat energy taken away by the blood is proportional to the perfusion rate. The magnitude of the transient temperature decreases with the blood perfusion rate. It is found from Fig. 1(a) that the temperatures obtained from the linear and non-linear DPL equations are very similar. However, this slight difference induces a more serious thermal damage, as shown in Fig. 1(b). It implies that thermal damage in living tissues is sensitive to temperature.

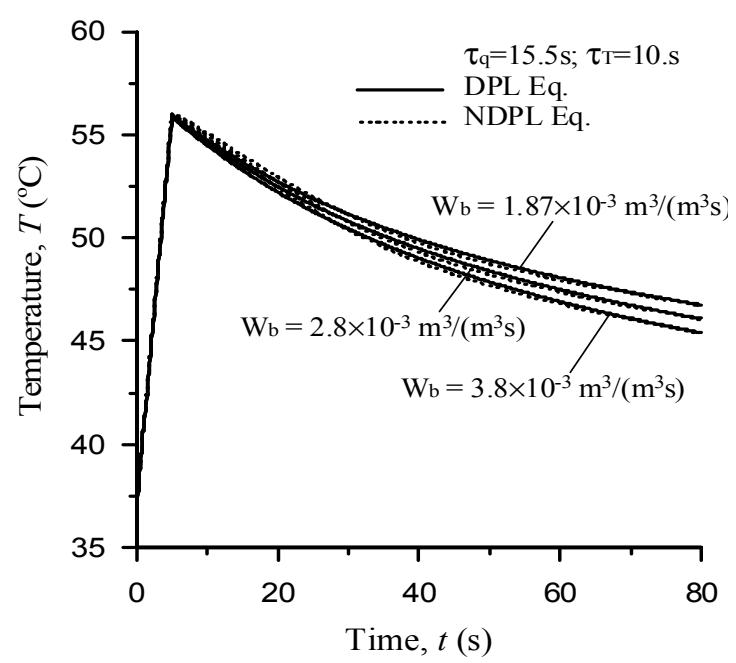

(a)

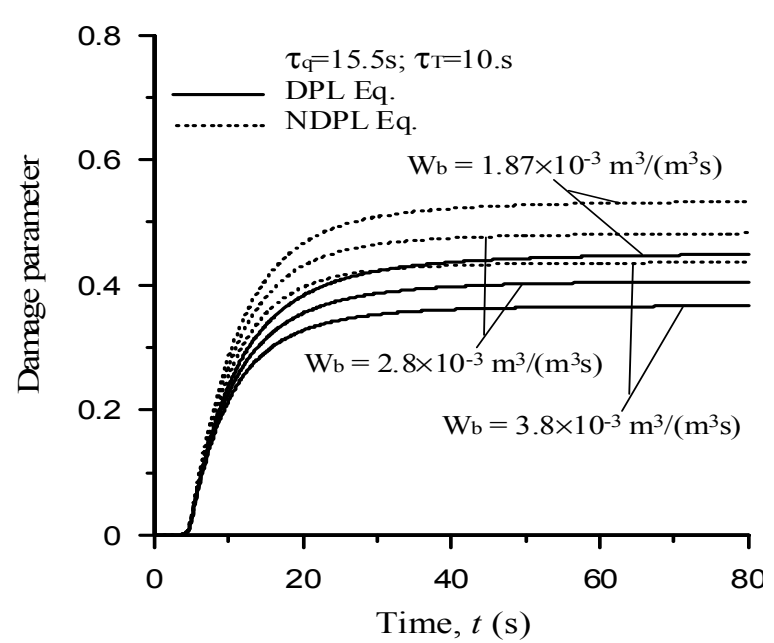

(b)

Fig. 1. Temperature and thermal damage at the irradiated surface for various blood perfusion rates.

The metabolic heat generation and blood perfusion are important characteristics of living tissues. As shown in Fig. 1, the blood perfusion is able to significantly affect the temperature rise during thermal treatment. The present work explores the effect of metabolic heat generation on the non-Fourier bio-heat transfer, as shown in Fig. 2, in which presents the temperature variation at the laser irradiated surface for $q_{m}=2.9 \times 10^{4} \mathrm{~W} / \mathrm{m}^{3}$ and $q_{m}=1.19 \times 10^{3} \mathrm{~W} / \mathrm{m}^{3}$. It is seen that the thermal effect is not obvious, and the temperature difference between $q_{m}=2.9 \times 10^{4} \mathrm{~W} / \mathrm{m}^{3}$ and $q_{m}=$ $1.19 \times 10^{3} \mathrm{~W} / \mathrm{m}^{3}$ is quite small. For the effects of of $\tau_{q}^{2}$ and $\tau_{T}^{2}$, the temperature predicted with the non-linear DPL equation is slightly larger than that with the linear DPL equation at the initial times. Correspondingly, the value of damage parameter based on the non-linear DPL equation is larger. 


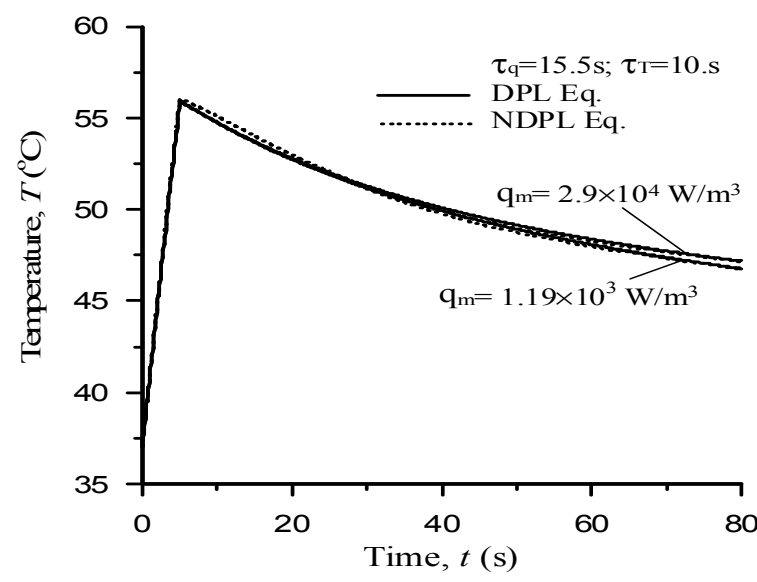

(a)

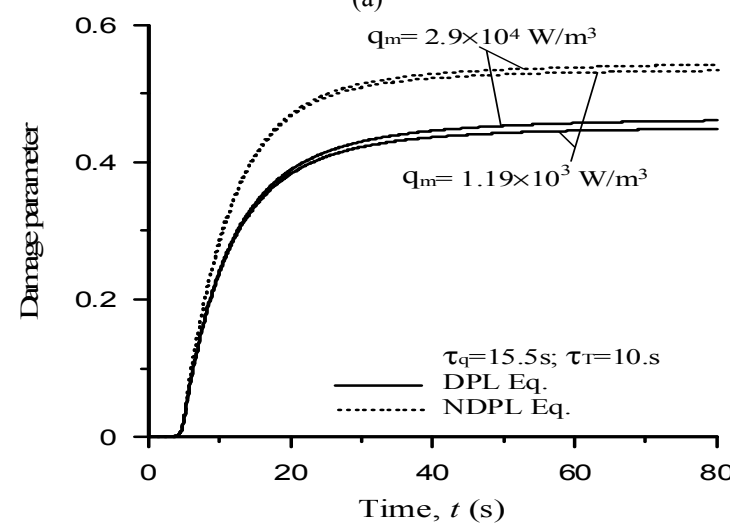

(b)

Fig. 2. Effects of metabolic heat generation on the temperature and damage parameter at laser irradiated surface.

\section{CONCLUSIONS}

A non-linear DPL equation of bio-heat transfer, which includes blood perfusion and metabolic heat generation, is numerically solved for thermal response, and to assess thermal damage to the tissue by a rate process equation. The comparison of temperature variation and the tissue damage parameter at the laser irradiated surface with those obtained from the linear dual-phase-lag equation is done. For the effects of $\tau_{q}^{2}$ and $\tau_{T}^{2}$, the non-linear DPL equation predicts a larger value of temperature during the laser heating. Although the difference between the temperatures obtained from the linear and non-linear DPL equations is slight, the difference in thermal damage is quite obvious. It implies thermal damage in living tissues is sensitive to temperature.

\section{REFERENCES}

[1] J. Liu, X. Chen, and L.X. Xu, "New thermal wave aspects on burn evaluation of skin subjected to instantaneous heating," IEEE. Trans. Biomedical Engineering, vol. 46, pp. 420-428, 1999.

[2] P.J. Antaki, "New interpretation of non-fourier heat conduction in processed meat," ASME J. Heat Transfer, vol. 127, pp. 189-193, 2005.

[3] D.Y. Tzou, Macro- to Microscale Heat Transfer: The Lagging Behavior, Taylor \& Francis, Washington, DC, USA, 1996.

[4] F. Xu, K. A. Seffen, and T. J. Lu, "Non-fourier analysis of skin biothermomechanics," Int. J. Heat and Mass Transfer, vol. 51, pp. 2237-2259, 2008.

[5] K. C. Liu and H. T. Chen, "Investigation for the dual phase lag behavior of bio-heat transfer," International Journal of Thermal sciences, vol. 49, pp. 1138-1146, 2010.

[6] K. C. Liu and C. T. Lin, "Solution of an inverse heat conduction problem in bi-layered spherical tissue," Numer. Heat Transfer A, vol. 58, pp. 802-818, 2010.

[7] D. Y. Tzou and W. Dai, "Thermal lagging in multi-carrier systems," International Journal of Heat and Mass Transfer, vol. 52, pp. 1206-1213, 2009.

[8] D. Y. Tzou, Z. Y. Guo, "Nonlocal behavior in thermal lagging," International Journal of Thermal Sciences, vol. 49, pp. 1133-1137, 2010.

[9] K. C. Liu, "Nonlinear behavior of thermal lagging in concentric living tissues with Gaussian distribution source," Int. J. Heat and Mass Transfer, vol. 54, pp. 2829-2836, 2011.

[10] J. Zhou, J. K .Chen, and Y. Zhang, "Dual-phase lag effects on thermal damage to biological tissues caused by laser irradiation," Computers in Biology and Medicine, vol. 39, pp. 286 - 293, 2009.

[11] A. Kanezaki, A. Hirata, S. Watanabe, and H. Shirai, "Parameter variation effects on temperature elevation in a steady-state, one-dimensional thermal model for millimeter wave exposure of ooneand three-layer human tissue", Phys. Med. Biol., vol. 55, pp. $4647-4659,2010$.

[12] C. M. Gardner, S. L. Jacques, and A. J. Welch, "Light transport in tissue: Accurate, heuristic expressions for one dimensional fluence rate and escape function based upon monte carlo simulations", Lasers in Surgery and Medicine, vol. 18, pp. 129-138, 1996.

[13] A. J. Welch, "The thermal response of laser irradiated tissue," IEEE Journal of Quantum Electronics, vol. 20, pp. 1471 - 1481, 1984.

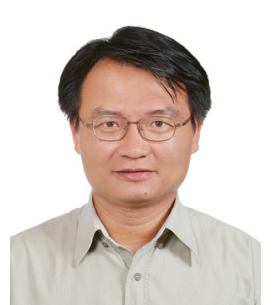

Kuo-Chi Liu received his B.S.AME degree from National Chung Hsing University, Taichung, Taiwan in 1983, M.S.AME degree from National Taiwan University, Taipei, Taiwan in 1985 and Ph.D.ME from National Cheng Kung University, Tainan, Taiwan in 2002. He is a professor in the department of Mechanical Engineering, Far East University. His current research interests are Bio-heat transfer, thermoelectric conversion technology, and pneumatic control. 